\title{
Insulin paradox, aging, bone health and growth in the context of mitochondrial function
}

Dear Editor,

Insulin paradox (as it is generally referred to) has recently been reported to show up in more than expected scientific investigations ${ }^{1-3}$. This puzzle has also involved studies in bone health and longevity. Increase in lifespan of mice and C. elegans knockouts of growth hormone receptor gene has opened an interesting area of research. To this effect, an interesting paper ${ }^{4}$ seems to integrate certain cardinal aspects of aging, bone health, and growth with mitochondrial function and insulin; indicating role for insulin signaling at several levels in differentiation, growth and maintenance of osteocytes and life span determination. It has been found that growth hormone receptor knockout (GHRKO) mice display increased insulin, insulin like growth factor-1 (IGF1), cytoplasmic/mitochondrial reactive oxygen species (ROS), life span and decreased mitochondrial membrane potential, glucose transporter-1 (GLUT-1) expression, steady state ATP, NADH redox index, glutathione, oxygen consumption rate, mitochondrial reserve capacity and skeletal health $\operatorname{span}^{4,5}$. Association of increased insulin and IGF-1 with extended life span is a novel finding seemingly not in agreement with previous reports ${ }^{6-9}$. An imperative facet of these findings is reduced mitochondrial volumetric density and mitochondrial intensity density in vivo with a parallel finding of no reduction in mitochondrial volumetric density and reduced mitochondrial intensity density in the primary osteocyte cultures ${ }^{4,5}$.

It could be argued that physical activity in mice (which may be conjectured to the phenomenon of exercise in humans) has much to do with skeletal dynamics and may affect mitochondrial volumetric density ${ }^{10,}{ }^{11}$ when viewed in

\begin{tabular}{l|l}
\hline & Access this article online \\
\hline & Website: \\
\hline
\end{tabular}

context of bioenergetic demands (in addition to the argument of osteocytes being deep buried and relatively less proliferative as suggested by Yakar et al., 2018 and Liu et al., $2019^{1,4}$ ). It appears that physical activity has a role to play in determining mitochondrial volume ${ }^{10-12}$ with a caveat that investigators have reported these findings in various cells (while this scenario with osteocytes has not been reported yet). As physical activity cannot be assessed in cell lines it would be worthwhile to study rodent/mouse models by subjecting them to various exercise practices ${ }^{13}$. Also, the elevation of insulin and IGF-1 in GHRKO mice and increased adiposity and insulin sensitivity (though the evaluation of the expression of insulin receptor in these experiments have not been explicated in comprehensive detail) is relevant. Change in insulin sensitivity can be interpreted in terms of hyperinsulinemia observed in these mice yet it may also be due to altered expression of insulin receptors (IR). So IR expression pattern may well be a parallel and presumably a cardinal biological feature in osteocyte health, pertinent to GHRKO mice. This rationale has important implications in clinical management of diabetes, bone health and metabolic syndrome. To add to this logic, insulin has been shown to improve mitochondrial function in a PI3K/Akt-dependent manner ${ }^{14}$ and increase in insulin levels might also be interpreted as a response (or associated factor) to alterations in mitochondrial volume density $^{15}$. Also, insulin prevents oxidative stress ${ }^{16}$ which

\section{Correspondence:}

Dr. Muneeb A Faiq, Post Doctoral Fellow

NYU Langone Eye Center, Department of Ophthalmology, NYU School of Medicine, NYU Langone Health, New York University, New York 10016, NY, USA.

Email:muneeb1983@gmail.com

How to cite this article: Qadri R, Sharique M, Pandit MA, Faiq MA. Insulin paradox, aging, bone health and growth in the context of mitochondrial function. jms [Internet]. 2019Mar.29 [cited 2019Apr.13];22(1). Available from: http://www.jmsskims.org/index.php/jms/article/view/373

Received:30-01-2019 Accepted: 25-03-2019 
Qadri R; et al; Insulin paradox, aging, bone health and growth

may be one of the mechanisms for prevention/delay of senescence/aging in the osteocytes presenting as extended life span.

Mitochondria have important role to play in insulin sensitivity $^{17}$. ROS formation may have maladaptive consequences that increase the rate of mutagenesis and stimulate proinflammatory processes. This provides a cogent rationale to evaluate inflammatory and immune aspects of GHRKO mediated bone impairments and osteocyte dysfunction. This may have implications in autoimmune disorders like rheumatoid arthritis (though the idea needs to be acknowledged with some caution). In addition, ROS, genetic factors, aging, and reduced mitochondrial biogenesis all contribute to altered expression of IRs. These factors contribute to changes in insulin signaling in classic and non-classic insulin target tissues. The prolonged life span and antiaging effect of GHRKO may also be explained in terms of insulin resistance mediated hyperinsulinemia (though the logic seems to run counterintuitive). On an additional note, mutations in Klotho have been associated with aging and bone loss. Why we assert Klotho is because its expression is controlled by insulin ${ }^{18}$. This integrates growth hormone receptor loss, mitochondrial dysfunction, insulin/IGF-1 elevation and IR expression into a single conceptual outlook. It is pertinent to mention that Klotho downplays insulin/IGF-1 pathway, Wnt pathway, TNF- $\alpha$, and TGF $\beta$ signaling thus acting as a co-receptor for FGF23 signal transduction. It also plays a role in sialidase activity mediated ion channel function ${ }^{19}$. It is interesting to note that Klotho polymorphisms have been associated with longevity and risk of disease in humans $\mathrm{s}^{20}$.

In the backdrop of all this, a coherent concept seems to emerge where bone health is being orchestrated by insulin signaling (bringing bone mineralization, trabecular spacing and, therefore, skeletogenesis, bone remodeling, mechanosensing and bone-cell crosstalk into a single picture). Experiments aimed at siRNA mediated knockdown or CRISPR mediated knockout of pivotal insulin signaling genes (like PI3K, IR, IGF-1, GSK-3 $\beta$ ) in osteocytes in culture and selected gene knockout in animals combined with experiments based on physical activity are likely to yield a wealth of information and suggest therapeutic measures in relevant bone diseases. Hence insulin paradox may appear as a biological conundrum at the outset but it presents an unexpected opportunity to be exploited for clinical applications in bone diseases, aging and endocrine health.

Thank you

\section{REFERENCES:}

1. Koshiyama H. Explanation of the Insulin Paradox From the Evolutionary Point of View. Jpn Clin Med. 2012;3:2124.

2. Cohen E, Dillin A. The insulin paradox: aging, proteotoxicity and neurodegeneration. Nat Rev Neurosci. 2008 Oct;9(10):759-67.

3. Ferris HA, Kahn CR. Unraveling the Paradox of Selective Insulin Resistance in the Liver: the BrainLiver Connection. Diabetes. 2016 Jun;65(6):14811483.

4. Liu Z, Solesio ME, Schaffler MB, Frikha-Benayed D, Rosen CJ, Werner H, Kopchick JJ, Pavlov EV, Abramov AY6, Yakar S. Mitochondrial Function Is Compromised in Cortical Bone Osteocytes of LongLived Growth Hormone Receptor Null Mice. J Bone Miner Res. 2019 Jan;34(1):106-122.

5. Yakar S, Werner H, Rosen CJ. Insulin-like growth factors: actions on the skeleton. Yakar S, Werner H, Rosen CJ. J Mol Endocrinol. 2018 Jul;61(1):T115T137.

6. Ma C, Tonks KT, Center JR, Samocha-Bonet D, Greenfield JR. Complex interplay among adiposity, insulin resistance and bone health. Clin Obes. 2018 Apr;8(2):131-139.

7. Diana van H. Insulin, IGF-1 and longevity. Aging Dis. 2010 Oct;1(2):147157.

8. Riia KJ, Edward OL, Darlene EB, John WM, John JK. The GH/IGF-1 axis in ageing and longevity. Nat Rev Endocrinol. 2013 Jun;9(6):366376.

9. Johnson JD, Templeman NM. Hyperinsulinemia Causes Age-Dependent Insulin Resistance and Reduces Lifespan. Canadian Journal of Diabetes. Oct 2016;40(5):S59S60

10. Meinild Lundby AK, Jacobs RA, Gehrig S, de Leur J, Hauser M, Bonne TC, Flück D, Dandanell S, Kirk N, Kaech A, Ziegler U, Larsen S, Lundby C. Exercise training increases skeletal muscle mitochondrial 
Qadri R; et al; Insulin paradox, aging, bone health and growth

volume density by enlargement of existing mitochondria and not de novo biogenesis. Acta Physiol (Oxf). 2018 Jan;222(1).

11. Ørtenblad N. Mitochondrial increase in volume density with exercise training: More, larger or better? Acta Physiol (Oxf). 2018 Jan;222(1).

12. van Ekeren GJ, Cornelissen EA, Stadhouders AM, Sengers RC. Increased volume density of peripheral mitochondria in skeletal muscle of children with exercise intolerance. Eur J Pediatr. 1991 Aug;150(10):744-50.

13. Holloszy JO, Smith EK, Vining M, Adams S. Effect of voluntary exercise on longevity of rats. J Appl Physiol (1985). 1985 Sep;59(3):826-31.

14. Ribeiro M, Rosenstock TR, Oliveira AM, Oliveira CR, Rego AC. Insulin and IGF-1 improve mitochondrial function in a PI-3K/Akt-dependent manner and reduce mitochondrial generation of reactive oxygen species in Huntington's disease knock-in striatal cells. Free Radic Biol Med. 2014 Sep; 74:129-44.

15. Morino K, Petersen KF, Dufour S, Befroy D, Frattini J, Shatzkes N, Neschen S, White MF, Bilz S, Sono S, Pypaert M, Shulman GI. Reduced mitochondrial density and increased IRS-1 serine phosphorylation in muscle of insulin-resistant offspring of type 2 diabetic parents. J Clin Invest. 2005 Dec;115(12):3587-93.

16. Faiq MA, Dada T. Diabetes Type 4: A Paradigm Shift in the Understanding of Glaucoma, the Brain Specific Diabetes and the Candidature of Insulin as a Therapeutic Agent. Curr Mol Med. 2017;17(1):46-59.

17. Kim J, Wei Y, Sowers JR. Role of Mitochondrial
Dysfunction in Insulin Resistance. Circ Res. 2008 Feb;102(4):401414.

18. Tracey B. Tucker Zhou, Gwendalyn D. King, CiDi Chen, and Carmela R. Abraham. Biochemical and Functional Characterization of the Klotho-VS Polymorphism Implicated in Aging and Disease Risk. J Biol Chem. 2013 Dec;288(51):3630236311.

19. Cha SK, Ortega B, Kurosu H, Rosenblatt KP, Kuro OM, Huang CL. Removal of sialic acid involving Klotho causes cell-surface retention of TRPV 5 channel via binding to galectin-1. Proc Natl Acad Sci USA. 2008; 105, 9805-9810.

20. Invidia L, Salvioli S, Altilia S, Pierini M, Panourgia MP, Monti D, De Rango F, Passarino G, Franceschi C. The frequency of Klotho KL-VS polymorphism in a large Italian population, from young subjects to centenarians, suggests the presence of specific time windows for its effect. Biogerontology. 2010; 11, 6773.

\footnotetext{
Rizwana Qadri', Mohd Sharique ${ }^{2}$, Muzafar A. Pandit ${ }^{3}$, Muneeb A. Faiq ${ }^{4}$

'Department of Laboratory Medicine, ${ }^{2}$ Center for Integrative Medicine and Research, ${ }^{3}$ Department of Pathology, AllMS, New Delhi

${ }^{4}$ NYU Langone Eye Center, Department of Ophthalmology, NYU School of Medicine, NYU Langone Health, New York University, New York10016, NY, USA.
} 